Cómo citar este artículo / Referencia normalizada

J Rúas-Araújo, F Campos-Freire, I Puentes-Rivera (2016): “Utilización y valoración de las redes sociales generalistas y buscadores bibliográficos en las universidades gallegas".

Revista Latina de Comunicación Social, 71, pp. 1.187 a 1.207

http://www.revistalatinacs.org/071/paper/1141/61es.html

DOI: $10.4185 / R L C S-2016-1141$

\title{
Utilización y valoración de las redes sociales generalistas y buscadores bibliográficos en las universidades gallegas
}

\section{Use and assessment of general-interest social networks and academic databases in Galician universities}

José Rúas-Araújo [CV] [ $\mathrm{DD}$ http://orcid.org/0000-0002-1922-9644]
[G http://scholar.google.es/citations?user=DLiqzqkAAAAJ\&hl=es]

Profesor titular de la Facultad de Ciencias Sociales y Comunicación. Universidad de Vigo, España joseruas@uvigo.es

Francisco Campos-Freire [CV] [ $\underline{\text { http://orcid.org/0000-0003-1831-1838] }}$

\footnotetext{
[G https://scholar.google.es/citations?user=feJKfd8AAAAJ\&hl=es]
}

Profesor titular de la Facultad de Ciencias de la Comunicación. Universidad de Santiago de

Compostela, España - francisco.campos@ usc.es

Iván Puentes-Rivera [CV] [

http://orcid.org/0000-0003-1982-0984]

[ G https://scholar.google.es/citations?user=6pZK8ecAAAAJ\&hl=es]

Investigador predoctoral de la Facultad de Ciencias Sociales y de la Comunicación. Universidad de Vigo, España - ivanpuentes@uvigo.es

\begin{abstract}
s
[ES] Introducción: Se analiza el conocimiento, uso, valoración e impacto de las redes sociales Facebook y Twitter y los buscadores bibliográficos por parte del Personal Docente e Investigador (PDI) de las tres universidades gallegas. Metodología: Se realizó una encuesta sobre el universo de los 5.498 docentes e investigadores, obteniendo una muestra de 463 respuestas, con un margen de error inferior al 5\% y un intervalo de confianza del 95\%. Resultados: El 50,2\% del PDI considera la idoneidad de Facebook y Twitter para la docencia y la investigación frente al 49,8\% que no. Scopus y Google Scholar son los buscadores más conocidos, con porcentajes superiores al $80 \%$ y una utilización superior al 60\%, y EBSCO y EMBASE los menos. Discusión y conclusiones:
\end{abstract}


Existe un estimable conocimiento y utilización de buscadores y opiniones enfrentadas entre quienes consideran la idoneidad de Facebook y Twitter para la docencia y la investigación y quienes no, lo cual sugiere un debate.

[EN] Introduction: This article examines the knowledge, use, assessment and impact of the social networks Facebook and Twitter and bibliographic databases among the Teaching and Research Staff (TRS) of three Galician universities. Methods: The study is based on a survey conducted among a sample of 463 respondents selected from a universe of 5,498 professors and researchers. The survey has a margin of error of $5 \%$ and a confidence level of $95 \%$. Results: $50.2 \%$ of the TRS considers that Facebook and Twitter are suitable for teaching and research while the remaining 49.8\% thinks otherwise. Scopus and Google Scholar are the most known and used databases, with percentages above $80 \%$ and 60\%, respectively, while EBSCO and EMBASE are the least known.

Discussion and conclusions: There is significant knowledge and use of academic databases, but the opinions regarding the suitability of Facebook and Twitter for teaching and research activities are very divided.

\section{Keywords}

[ES] redes sociales; buscadores bibliográficos; universidades; ORCID; marcadores sociales; perfiles y herramientas digitales científicas.

[EN] Social networks; academic search engine; universities; ORCID; social bookmarking; scientific digital profiles and tools

\section{Contents}

[ES] 1. Introducción. 2. Uso docente de las redes sociales. 3. Método. 4. Resultados. 4.1. Utilización y valoración de Facebook y Twitter. 3.2. Utilización y valoración de buscadores. 5. Discusión y conclusiones. 6. Notas. 7 .Referencias bibliográficas.

[EN] 1. Introduction. 2. 2. Educational use of social networks. 3. Methods. 4. Results. 4.1. Use and assessment of Facebook and Twitter. 4.2. Use and assessment of search engines. 5. Discussion and conclusions. 6. Notes. 7. References.

\section{Introducción}

De año en año cambian sus caras, su fisonomía, vestimenta, estilismo y también las herramientas que acompañan las carpetas y las mochilas de los estudiantes en las distintas aulas de las Universidades españolas o de cualquier parte del mundo occidental. Ya son menos los cuadernos, los bolígrafos y los libros en papel que los alumnos llevan a clase cada día. Como son muchas menos también las lecturas y consultas que realizan sobre documentos en papel, frente al recurso masivo e instantáneo de recurrir al buscador de Google o a la Wikipedia. Este es el cambio en los usos de las herramientas didácticas que se aprecia a primera vista en las aulas de los últimos tres o cuatro años.

No han cambiado, sin embargo, las luces del techo o las persianas de las ventanas de las aulas, las mesas y sillas -deterioradas algunas de ellas- ni tampoco los encerados o las tizas que aún ilustran las exposiciones de parte del profesorado. Sin embargo, el ambiente informático y conectivo 
personal que domina entre el alumnado es bien distinto. Predominan los ordenadores portátiles, las tabletas y los teléfonos móviles inteligentes. Y a través de la mayor parte de esos dispositivos conectados ellos combinan el seguimiento dactilográfico de las exposiciones de los profesores con la atención a los mensajes de las redes sociales abiertas de sus amigos o agregados.

Muchos alumnos mantienen al mismo tiempo conectadas sus redes digitales e interaccionan en ellas mientras el profesor desarrolla la clase magistral. Algunos de ellos simultanean la toma de notas en su pantalla principal mientras tienen abiertas o minimizadas otras dos o tres ventanas más correspondientes a Facebook, Twitter, Instagram o Snapchat. El uso multipantalla no sólo ya es propio del consumo de medios de comunicación por parte del público joven sino también de muchas de sus otras prácticas sociales cotidianas, entre ellas la de la clase y el estudio. La atención estudiantil a la conversación virtual de las redes se remezcla e hibrida con las teorías y conceptos de las clases en una especie de "collage", "remix" o "mash-up" didáctico comunicacional (Manovich, 2007).

Ante este nuevo paisaje discente de nuestras aulas, la reacción por parte del claustro docente oscila entre el desconcierto, incapacidad, impotencia, prohibición, rechazo, histeria, nerviosismo, inhibición, pasividad o búsqueda de estrategias alternativas para recuperar la totalidad de la atención mediante la incorporación de esas nuevas herramientas al ritmo pedagógico. No es fácil hacerlo ni enfrentarse a esa nueva realidad tecnológica -salvo que se opte por la solución radical de la inhibición técnica de las aulas, es decir, espacios libres de wifi- porque el reto de la sociedad del aprendizaje (Stigliz y Greenwald, 2016) en la que estamos inmersos tampoco puede desentenderse ni descontextualizarse del ambiente conectivo en el que viven nuestros discípulos.

El profesorado, como la mayoría de las personas, asume que las redes sociales digitales son una nueva forma de conectividad de la sociabilidad actual. Si cada uno de nosotros estamos agregados y empleamos las redes con mayor o menor asiduidad en nuestra vida cotidiana, no tenemos argumentos lógicos para llevarnos las manos a la cabeza por la fruición con que las usan nuestros alumnos. Lo que todavía no sabemos bien es cómo utilizarlas e implementarlas adecuadamente en las relaciones con los estudiantes. No sólo como parte de la didáctica sino también de la tutoría, de la investigación científica y de la relación global con la sociedad a la que nos debemos por nuestra función social de mediadores del conocimiento y el aprendizaje.

Partiendo de esas premisas, el objeto de esta investigación es conocer cómo usan y valoran las redes sociales generalistas - las que ocupan a nuestros alumnos en las aulas, en la calle y en sus casas- los profesores de la Universidad. En este caso el objeto de estudio no son específicamente las redes temáticas científicas, donde se encuentran muchos profesores y pocos estudiantes aún, sino las de carácter generalista a través de las que se conectan, conversan e interactúan nuestros alumnos. También hemos querido saber cómo y en qué medida usan los profesores otras herramientas digitales, como son los buscadores generalistas o especializados y otros instrumentos de gestión de la información.

La investigación se contextualiza con los datos cuantitativos -recabados entre enero y abril del presente curso académico- de seguidores de las tres universidades objeto de estudio en las principales redes y con la opinión institucional sobre el tema de los representantes de los respectivos vicerrectorados y áreas de formación e innovación educativa de cada universidad y los datos sobre los cursos de formación ofertados. Esos son los objetivos de esta investigación, que abarca a un colectivo de más de 5.000 profesores de las Universidades de Galicia, cuyos resultados bien podrían representar un prototipo medio del actual estamento docente español. La cuestión es que las redes sociales digitales están cada vez más presentes en la sociedad, en las universidades y 
nuestras clases, pero ¿las aprovechamos adecuadamente o nos vemos envueltos por ellas y tratamos de navegar como podemos para no naufragar?.

\section{Uso docente de las redes sociales}

La observación directa del comportamiento de los alumnos nos permite constatar un uso intensivo lúdico, relacional e informativo- de las redes sociales generalistas, así como una tímida introducción de las mismas en las relaciones docentes o prácticas de los profesores con sus estudiantes, a pesar de la aceptación de su importancia. El estado de la investigación constata esta misma impresión, a partir del reconocimiento de la conectividad social, su interés de cara a la inserción profesional, el respaldo esencial en la educación a distancia, la divulgación de nuevas experiencias de las redes en las aulas, la apertura de las compuertas de la ciencia abierta, el uso de las redes académicas, la aparición de nuevas métricas científicas y la utilidad de los identificadores digitales tipo ORCID.

El modelo colaborativo y abierto de las redes sociales es toda una revolución dentro de las estructuras formales o "rígidas torres de marfil" del sistema universitario más tradicional que presenta una gran oportunidad para una relación más horizontal, dinámica e intensiva entre profesores y alumnos en nuevos entornos que enriquecen la formación contemporánea de lo simbólico (Georgescu y Popescul, 2014; Núñez-Gómez y García-Guardia, 2010). Esta conectividad social entre profesores y alumnos (Bhagat, Wun y Chang, 2016) permite reducir la distancia en la percepción formal de los docentes, más allá de sus clases magistrales y del sistema convencional de evaluaciones, estableciendo una "tercera vía para reforzar el trabajo real a través de una nueva relación intelectual" (Carney, 2010), en línea con los nuevos sistemas pedagógicos y las propias recomendaciones del Sistema del Espacio Educativo Europeo (EEES).

Los profesores más inquietos e innovadores introducen las redes no sólo para comunicarse con sus alumnos sino también para captar su atención en clase, que es una de las grandes preocupaciones y problemas en la situación actual. A través de Twitter y teléfonos móviles algunos docentes proponen escribir en las pizarras electrónicas o en sus presentaciones en power point preguntas de respuesta inmediata a la cuenta del profesor, que se evalúan según la rapidez de cada retweet, para resolver el problema de falta de concentración o de distracción con respecto a otras redes (Kim, Jeong, Ji et al., 2015). Son experiencias pioneras e innovadoras, porque los resultados de las investigaciones realizadas (Viassone, 2014) muestran que los estudiantes utilizan las redes preferentemente para interactuar con sus compañeros y profesores.

Por razones obvias, la introducción del uso de las redes es más intensiva en la educación a distancia y en la transformación de la figura del profesor en monitor o tutor de comunidades de aprendizaje del elearning (Dumitrache, Gheorghe y Moldovan, 2014). En la enseñanza presencial y en el ámbito general esas necesidades de aplicación de nuevas herramientas han generado demandas poco atendidas de alfabetización digital (Georgescu y Popescul, 2014) y de creación de redes educativas temáticas para la formación del profesorado (Díaz, Vázquez-Martínez y McMullin, 2014). Las iniciativas en estos aspectos han sido más particulares y voluntaristas que generales e institucionales.

Sin embargo el incremento de herramientas digitales y propagación de MOOC (Massive Open Online Course) ha sido exponencial en los últimos años; como lo es el uso de vídeos de YouTube o simuladores didácticos (Sergio, Navarro y Bernal, 2014), tanto en la enseñanza reglada como en las cotidianas prácticas autodidactas de esta nueva era sociotécnica en la que las personas hibridan aprendizaje y experiencias a través de las tecnologías disponibles (Kubatova, 2012). Podríamos decir también que los planes y las prácticas educativas institucionales se han visto desbordadas por la penetración de las nuevas tecnologías de la información y la comunicación. 
Del mismo modo las redes sociales digitales se han impuesto como un complemento importante para la formación de los perfiles profesionales para la futura inserción laboral de los estudiantes, ya que cada vez son más utilizadas por los reclutadores en el proceso de selección de personal (Roulin y Bangerter, 2013). En algunas investigaciones realizadas recientemente sobre las relaciones laborales y profesionales (Zhitomirsky-Geffet y Bratspiess, 2015) se percibe la utilidad de Facebook y Linkedin de forma muy similar, aunque con mayor efectividad de la segunda para la búsqueda de empleo. Como lo son para los profesores las redes académicas de difusión de las investigaciones propias y de seguimiento de las ajenas, juntamente con otras herramientas de utilidad para la docencia e investigación como los buscadores, los identificadores digitales de identidades (ORCID, Open Researcher and Contributor ID, y DOI, Digital Object Identifier) o los indicadores de reputación científica.

Las redes sociales científicas son plataformas de metamedios (medios informáticos y de comunicación evolucionados) que funcionan como ecosistemas de interacción mediante perfiles públicos o semipúblicos de afiliación para la difusión de las propias publicaciones y seguimiento de las de otros colegas, acceso abierto a millones de artículos y trabajos, curación de contenidos científicos, interconexión con constelaciones de aplicaciones de filtrado y búsquedas, desarrollo de conversaciones y relaciones de colaboración para investigaciones, aplicación de dispositivos de inteligencia artificial semántica, sistemas de crowdreview y socialreview, ofertas de empleo docentes, gestión de la identidad digital y formación de rankings e indicadores de reputación académica, así como de otros nuevos metaservicios digitales (Boyd y Ellison, 2007; Campos, 2015).

La gestión de la información científica fue pasando de los archivos estáticos de las bibliotecas a los repositorios digitalizados así como a los buscadores en red y de estos a las herramientas de administración de referencias (EndNote, Zotero, CiteULike), a los identificadores de objetos e identificadores digitales (DOI, creado en 2000 y ORCID, en 2012), a las almetrics o nuevas métricas de los datos (Álvarez-García, Gértrudix-Barrio y Rajas-Fernández, 2014), a las redes sociales digitales generalistas (MySpace, Facebook, Flickr, Youtube, Twitter) que se desarrollan entre 1999 y 2006 y a otras más especializadas y temáticas (LinkedIn en 2003, Mendeley, ResearchGate y Academia.edu, las tres últimas creadas en 2008).

Las bases de datos bibliográficas surgen de la gestión concentrada de los catálogos de revistas científicas y especializadas para su comercialización directa a las Universidades y bibliotecas de todo el mundo, que inicia EBSCO (Elton B. Stephens Company) en 1944 en Alabama (Estados Unidos), y secunda EMBASE del grupo holandés Elsevier, con 28 millones de registros de 8.400 revistas editadas desde 1947 sobre medicina o farmacología. Esta última fue vendida en 2010 al grupo norteamericano Omnicom. También conviven con bases de datos y servicios en línea gestionados por instituciones nacionales, como MEDLINE y PubMED, de la Biblioteca Nacional de Medicina de Estados Unidos.

Los dos gigantes mundiales que controlan la mayor parte de las publicaciones y revistas científicas son el grupo norteamericano Thomson Reuters y el holandés Elsevier. Ambos han tratado de combinar la transición del modelo impreso al digital, conservando sus propias plataformas de distribución de la información especializada. Thomson es el grupo matriz del servicio en línea de información científica ISI Web of Knowledge (WOK), que agrupa en su Web of Science (WOS) sus grandes bases de datos bibliográficas de 8.700 revistas de ciencia, tecnología, ciencias sociales, artes y humanidades así como más de cien mil actas de conferencias y congresos (ISi Proceedings) desde 1945. La cobertura de Scopus, del grupo Elsevier, se remonta a 1966 e integra 22.000 títulos de más de 5.000 editores. 
La filosofía de las bases de datos especializadas se traslada con toda su intensidad a Internet a mediados de la década de 1990 a través de la creación de buscadores: Alexa de Amazon (1996), Ask.com (1996), Sohu de China (1996), Trovator de España (1996), Yandex de Rusia (1997), PubMED (1997), Google Search (1997), MSN Search (1999, luego Yahoo! Search y fusión con Bing), Baidu de China (2000), Cuil (2006), entre otros. Las tres cuartas de las búsquedas globales de internet (en el Espacio Económico Europeo el 90\% según la CEE, 2016) son acaparadas por Google, que ha atacado también la especialización de los buscadores de WOS y Scopus mediante la adopción de un indicador propio (el índice H de Jorge Hirsch, en 20059 de reconocimiento de la reputación científica (De Pablos, Mateos y Túñez, 2013). Google usa 200 factores en el algoritmo de clasificación aunque su jerarquización prioriza Page Rank (PR), título, abstract y URL, lo que impone estrategias personales e institucionales de SEO para una adecuada gestión (Cheng-Jye et alt., 2016). Luego, la red social científica ResearchGate, creada en 2008, introduce también otro nuevo indicador, el RGScore, para evaluar la popularidad y calidad de las interacciones de sus artículos científicos.

La competencia que sufren las bases de datos y los buscadores especializados de WOS y Scopus, a pesar de sus catálogos exclusivos de revistas y los sistemas tradicionales de indicadores de reputación, les exigen evolucionar al ritmo de la innovación tecnológica, introduciendo nuevos servicios de alertas continuas, gestión de perfiles, monitores didácticos, sistemas de identificación digital propios (ResearcherID de WOS en 2008) o adoptados por Scopus (Open Researcher and Contributor ID, 2012), herramientas de gestión de la información (como EdNote de Thomson) o adquisición en 2013 de la red social Mendeley, creada en 2007 por parte de Elsevier.

El uso y gestión del espacio digital en el ámbito docente requiere no sólo herramientas sino también estrategias y metalenguajes propios. Son recursos y competencias que, en mayor o menor medida, se están incorporando tanto a la academia como a la vida social ordinaria. La gestión de perfiles, identidades, metadatos, buscadores y marcadores sociales digitales son aspectos fundamentales que no siempre se administran de la forma más eficiente.

No se conocen datos estadísticos globales precisos, pero la mayor parte del conocimiento en el mundo actual se gestiona a través de recursos digitales y el impulso de la llamada ciencia abierta o ciencia 2.0 es cada vez más fuerte (Nosek, 2012; Ortega, 2015; Tanksalvala, 2016). Algunas investigaciones recientes (Jamili y Nabavi, 2015) sostienen que el 61\% de las búsquedas académicas en Google corresponden a artículos de acceso abierto, especialmente en el ámbito de las ciencias de la vida. Otra investigación de la Universidad de Colorado (EE.UU.) demuestra empíricamente que los artículos publicados en revistas de acceso abierto obtienen más citas que los de publicaciones cerradas (Barker, Johnson, Michaelson, Stucky, 2014).

El debate sobre el concepto de ciencia abierta está directamente vinculado a la gestión de la propiedad intelectual, la digitalización del conocimiento, sus modelos de negocio y las tecnologías de innovación disruptiva (Christensen, Raynor y McDonald, 2015) que introduce internet y la web 2.0. Las revistas científicas acreditan los "colegios invisibles" (Crane, 1972) de difusión del conocimiento mediante sistemas de acreditación de la reputación, basados en evaluaciones de pares especializados y métricas de reputación. Alrededor de ese sistema se levantan grandes grupos editoriales de gestión del conocimiento (Thomson y Elsevier, más de 20.000 millones de dólares de facturación anual) de revistas, bases de datos, indicadores de reputación, buscadores y redes digitales. Bases de datos existen cientos de ellas pero su relevancia y utilidad está directamente relacionada con la propiedad y especialización en el acceso a los datos. 
La digitalización de archivos e interconectividad de los mismos es el primer eslabón que se rompe en la cadena de valor del modelo de negocio cerrado de gestión del conocimiento. El modelo de propiedad intelectual, basado en el copyright del editor y la patente del inventor, deja de ser eficiente porque la innovación digital (Kotsemir y Meissner, 2013) logra saltar la barrera de la accesibilidad sin protocolos de control que mantengan su monetización tradicional, en función de la que las empresas de revistas y bases de datos conservan las suscripciones de pago. La disrupción de esos modelos se acelera mediante la cultura colaborativa de la convergencia digital (Jenkins y Deuze, 2008) y las herramientas de la Web 2.0 que liberan millones de archivos digitales, dando paso a nuevos modelos abiertos como la Wikipedia o las redes sociales científicas.

El uso adecuado de perfiles e identidades digitales aumenta la visibilidad e intercambio de ideas entre los académicos e investigadores. La mayoría de los investigadores todavía no gestionan de forma eficiente, cuidadosa, actualizada y completa sus perfiles académicos según un estudio de la Universidad de Bergen (Mikki, Zygmuntowska, Gjesdal et alt., 2015). Los estudios sobre la inteligencia colectiva y la búsqueda social apuntan a los patrones de comportamiento y a las taxonomías centradas en el usuario (McDonnell y Siri, 2011). Esa información es la base sobre la que se articula la arquitectura de la metacomunicación científica. Los mecanismos que permiten esa relación de accesibilidad son los repositorios, las bases de datos, los buscadores y las redes de valor o reputación que intervienen en la gestión inteligente de la información.

Por obsolescencia, falta de interoperabilidad y necesidad de mayor convergencia, no existe todavía un método unificado ni unánimemente aceptado sobre los identificadores de metadatos bibliográficos pero en los últimos años se ha avanzado bastante. Los sistemas de DOI (identificador de trabajos digitales) y ORCID (código alfanumérico único de representación de cada autor) ayudan a todas las partes interesadas en las comunicaciones científicas a mejorar sus flujos de trabajo (Gasparyan, Akazhanov y Voronov, 2014; Mazov y Gureev, 2014).

El registro de ORCID, a pesar de su desconocimiento en algunas áreas, está creciendo rápidamente y resolviendo muchos problemas de ambigüedad o confusión con los nombres de los autores, a la vez que permite a las búsquedas muchos más cruces de disciplinas, organizaciones y países (Haak, Fenner, Paglione et al., 2012). Un estudio de Viviana Fernández-Marcial y Llarina González-Solar (2015) sobre el uso de herramientas de gestión de la identidad digital de los investigadores de la Universidad de Coruña a través de ORCID, ResearcherID, Scopus, Google Scholar, Researchgate y Mendeley revela que, a pesar de que los resultados no son totalmente negativos, los niveles deben mejorarse.

Las redes y cadenas de valor de la ciencia actual (Kothandaraman y Wilson, 2001; Allee, 2009; Peppard y Rylander, 2006) se activan a través de algoritmos de reputación e impacto (tradicionales y nuevas métricas generadas por indicadores de citación, visibilidad y popularidad). La conectividad y propagación de las redes digitales, tanto generalistas como temáticas, contribuye a ampliar la visibilidad, el conocimiento, la reputación, el número de citas y otras nuevas métricas como las del twimpact (Eysenbach, 2011). Lo que no puede excluir este sistema reticular de inteligencia digital es la necesidad de la ética y la transparencia frente a los riesgos y prevenciones de la manipulación humana o algorítmica por parte de nuevos actores robóticos posthumanos (Gourlay, 2014).

La explosión de las redes y herramientas sociales digitales, que nuestros alumnos experimentan con intensidad en sus relaciones interpersonales, no son todavía una práctica generalizada ni en las aulas ni en las relaciones con los profesores, a pesar de que se empieza a demostrar de que su uso refuerza el compromiso académico (Topaloglu, Caldibi y Oge, 2016). Esta investigación constata que los 
profesores usan las redes para la interconexión con sus pares, para promocionar su perfil de investigadores, para conocer otros autores y para la búsqueda académica pero mucho menos para la interacción con los alumnos. Los estudiantes, por su parte, son favorables al uso de las redes sociales para los propósitos educativos (Lim y Richardson, 2016).

Otra amplia investigación reciente del mismo tipo realizada en Italia (Manca y Ranieri, 2016) revela que el uso de los medios sociales es todavía bastante limitado y restringido en la educación universitaria y que los académicos no son muy proclives a integrar estos dispositivos en sus prácticas por varias razones, tales como la resistencia cultural, las cuestiones pedagógicas o las limitaciones institucionales. Existen diferencias entre los académicos en las formas que utilizan los medios sociales o los perciben, sobre todo en función de la disciplina científica a la que pertenecen. En general, los resultados hacen hincapié en las actitudes ambivalentes hacia los beneficios y retos de los medios sociales en el contexto de la educación, prevaleciendo los obstáculos sobre las ventajas.

Cuatro competencias y atributos esenciales para la enseñanza y aprendizaje en el siglo XXI son: a) adaptación a los cambios frecuentes y situaciones inciertas del mundo actual; b) colaboración y comunicación en entornos descentralizados; c) generación de datos y gestión de la información; y d) liberación del control de los sistemas mediante la exploración (Barak, 2016). La inmersión de nuestras Universidades en la inteligencia colectiva y las nubes de conocimiento no puede ser sólo una metáfora sino también una realidad inaplazable.

Con esta revisión de la literatura científica y de las investigaciones más recientes tratamos de reforzar la pertinencia de las observaciones e interrogantes que se plantean al principio de este artículo y que motivan las preguntas de la encuesta cuyos resultados se ofrecen a continuación.

\section{Método}

Esta encuesta forma parte de una investigación más amplia para conocer la penetración real, uso, popularidad, valoración e impacto de las redes sociales digitales generalistas y académicas en las tres universidades gallegas.

Para la realización de la encuesta se repartió un cuestionario online al conjunto de los investigadores y profesores de las tres universidades gallegas, a través de las listas de distribución interna de correo electrónico, y de los servidores y administradores de las cuentas y listados del PDI de cada universidad, tras cursar la correspondiente solicitud a los respectivos responsables y garantizar tanto la inclusión de los datos e identidad de los promotores de la investigación como el tratamiento anónimo de las respuestas obtenidas.

La encuesta se realizó al Personal Docente e Investigador (PDI), compuesto por funcionarios (catedráticos y profesores titulares), personal fijo y contratado laboral de las tres universidades gallegas, un total de 5.498 docentes e investigadores distribuido del siguiente modo: 2.099 personas de la USC, 1.983 de la UVigo y 1.416 de la UDC.

El total de las respuestas obtenidas fue de 463 personas, repartidas del siguiente modo: Universidad de Vigo, 173 respuestas (37,4\%), Universidad de Santiago de Compostela, 165 respuestas (35,6\%) y Universidad de A Coruña, 125 respuestas (27\%). La edad media de los docentes e investigadores que respondieron a la encuesta es de 46,58 años $\mathrm{y}$, mayoritariamente, fueron hombres los que respondieron $(55,3 \%)$. 
Con estos resultados, el margen de error obtenido por la aleatoriedad de la muestra es inferior al $5 \%$, con una precisión global del 4,328\%, teniendo en cuenta el número total de profesores e investigadores de las tres universidades gallegas y suponiendo que en la peor situación la probabilidad de respuesta en algún ítem de la encuesta sea de un 50\%, con un intervalo de confianza del $95 \%$.

\section{Resultados}

\section{a. Utilización y valoración de Facebook y Twitter}

Antes de presentar y explicar los resultados hay que matizar también la distinción entre redes de relaciones y de conocimiento (Zhang, Wu y Zhao, 2016). Las primeras corresponden más a la categoría generalista (Facebook y Twitter) y las segundas a las académicas o científicas (Researchgate y Academia). Las generalistas son más útiles para las relaciones de comunicación y marketing de las instituciones mientras que las científicas se orientan al apoyo y reputación de la investigación. En función de su orientación y enfoque se deben trazar y entender las estrategias.

Una primera aproximación sobre el seguimiento de Facebook y Twitter en la Universidad de Santiago de Compostela (USC), la Universidad de A Coruña (UDC) y la Universidad de Vigo (UVigo) muestra un respaldo relativo por parte del conjunto de la comunidad universitaria (alumnado, profesorado, investigadores y personal de administración y servicios), teniendo en cuenta incluso el hecho de que a estas cuentas pueden agregarse personas ajenas a cada institución (esto justificaría que el porcentaje de seguidores de la cuenta de Twitter de la UVigo sea mayor que el número de integrantes de su comunidad universitaria). La única de las tres universidades que muestra un porcentaje estimable de seguidores en Twitter y entre personas a las que le gusta y siguen su Facebook es la de A Coruña, circunstancia que también podría derivarse de la existencia, en el caso de la USC y la UVigo, de dos cuentas, una de usuarios (no oficial) y otra institucional.

Tabla 1. Las universidades gallegas en Facebook y Twitter [1]

\begin{tabular}{|l|l|l|l|l|}
\hline & $\begin{array}{l}\text { Twitter. } \\
\text { No. Seguidores }\end{array}$ & $\begin{array}{l}\text { \% sobre total } \\
\text { comunidad }\end{array}$ & $\begin{array}{l}\text { Facebook } \\
\text { No. Me gusta }\end{array}$ & $\begin{array}{l}\text { \% sobre total } \\
\text { comunidad }\end{array}$ \\
\hline USC & 6.083 & $20,56 \%$ & 4.290 & $14,50 \%$ \\
\hline UDC & 24.497 & $95,17 \%$ & 15.653 & $60,81 \%$ \\
\hline UVigo & 27.666 & $116,53 \%$ & 7.325 & $30,85 \%$ \\
\hline
\end{tabular}

Fuente: Elaboración propia

Por otra parte, la opinión generalizada, expresada desde los vicerrectorados de profesorado y comunicación de las tres universidades gallegas consultados, es que la incorporación de las redes sociales generalistas y académicas a la docencia y la investigación debe constituir un tema estratégico y, en consecuencia, incentivarse como elemento innovador, independientemente de la voluntad de los docentes, y que, además, deben aprovecharse todas las posibilidades que ofrece el mercado para poder mejorar la calidad de la docencia y la investigación, siempre y cuando aporten un valor positivo a su mejora. 
En cuanto a la oferta realizada en este sentido, las tres universidades gallegas plantearon, en los últimos tres años, algunos cursos, a través de sus programas y unidades de formación e innovación educativa (PFID, UFA y FIE, en el caso de la USC, UDC y UVigo, respectivamente), que muestran cierto interés inicial en la promoción de la utilización de las redes sociales generalistas y académicas. En concreto, tanto la USC como la UVigo ofrecieron sendos cursos sobre aprendizaje en red y las aplicaciones educativas el empleo de herramientas de la web social en el desarrollo de entornos personales de aprendizaje (PLE) y, además, la UDC organizó un curso específico sobre utilización de las redes sociales científicas y la gestión de referencias bibliográficas. Una oferta complementada a través de los cursos sobre recursos bibliográficos promovidos, periódicamente, desde el servicio de biblioteca de las tres universidades. Sin embargo, también es llamativo que esta oferta de las universidades gallegas tuviese una acogida relativa por parte de la comunidad universitaria, a juzgar por el escaso número de matriculados en la mayoría de los cursos.

Pero entrando ya en los resultados concretos de la encuesta realizada, indicar que las primeras cuestiones planteadas a los docentes e investigadores consultados eran si consideraban adecuado el empleo de las redes sociales generalistas Facebook y Twitter para la docencia y la investigación. Las respuestas fueron bastante igualadas entre partidarios y detractores, en concreto, un 50,2\% de los encuestados (225 personas) las consideran adecuadas, frente al 49,8\% (223 personas) que apuntó que no.

Tabla 2. ¿Considera Facebook y Twiter para la docencia y la investigación?

\begin{tabular}{|l|l|l|l|l|l|}
\hline \multicolumn{2}{|c|}{} & Frecuencia & Porcentaje & $\begin{array}{l}\text { Porcentaje } \\
\text { válido }\end{array}$ & $\begin{array}{l}\text { Porcentaje } \\
\text { acumulado }\end{array}$ \\
\hline \multirow{2}{*}{ Válidos } & $\mathrm{Si}$ & 225 & 48,6 & 50,2 & 50,2 \\
\cline { 2 - 6 } & No & 223 & 48,2 & 49,8 & 100,0 \\
\cline { 2 - 6 } & Total & 448 & 96,8 & 100,0 & \\
\hline Perdidos & Sistema & 15 & 3,2 & & \\
\hline Total & 463 & 100,0 & & \\
\hline
\end{tabular}

Fuente: Elaboración propia

De las tres universidades gallegas, es la UVigo la que muestra un mayor porcentaje de docentes e investigadores que consideran positivo el uso de Facebook y Twitter para la docencia y la investigación (55,6\%), frente al 44,4\% de los miembros de esta institución que no consideran el uso de estas herramientas. Por su parte, la UDC es la que muestra un mayor porcentaje y diferencia, entre el PDI en contra de la utilización de estas redes sociales $(61,3 \%)$ y a favor $(38,7 \%)$. La USC es la institución donde las opiniones se encuentran más igualadas y enfrentadas, entre partidarios $(53,2 \%)$ y detractores $(46,8 \%)$ de la utilización de estas redes sociales para la docencia y la investigación.

Por categorías profesionales, puede observarse que, en líneas generales, son los colectivos más asentados laboralmente los que se muestran más en contra del empleo de Facebook y Twitter para la docencia y la investigación, a excepción de los catedráticos de universidad, donde la proporción de partidarios y detractores es casi idéntica. En concreto, el $57,1 \%$ de los profesores contratados doctores, el 55,6\% de los titulares y el 48,9\% de los catedráticos de universidad no consideran la utilización de estas redes sociales para la docencia y la investigación. 


\section{Gráfico 1. Consideración sobre uso de Facebook y Twitter}

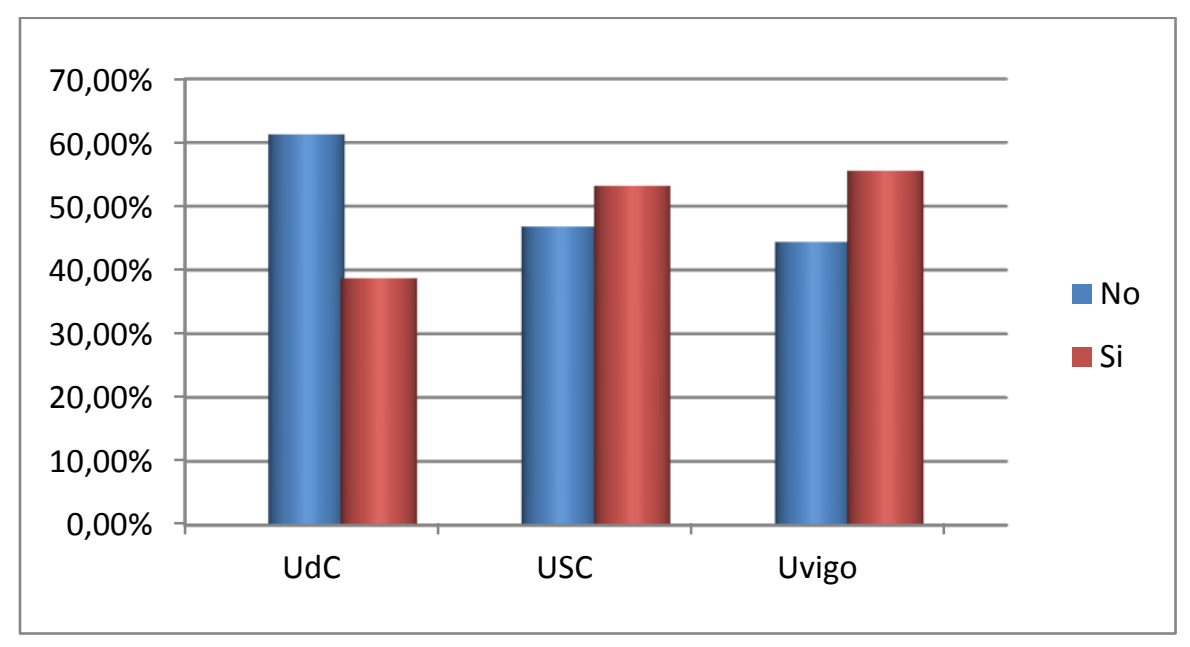

Fuente: Elaboración propia

Por el contrario, el mayor número de partidarios del empleo de estas herramientas para usos docentes e investigadores se encuentra en los investigadores predoctorales $(72,5 \%$ a favor frente al $27,5 \%$ en contra), profesores asociados $(65,8 \%$ a favor frente al $34,2 \%$ en contra), ayudantes doctores $(57,9 \%$ a favor y $42,1 \%$ en contra) e investigadores postdoctorales $(56,3 \%$ a favor y $43,8 \%$ en contra), tal y como puede observarse en el siguiente gráfico.

\section{Gráfico 2. Consideración sobre uso de Facebook y Twitter por categorías profesionales}

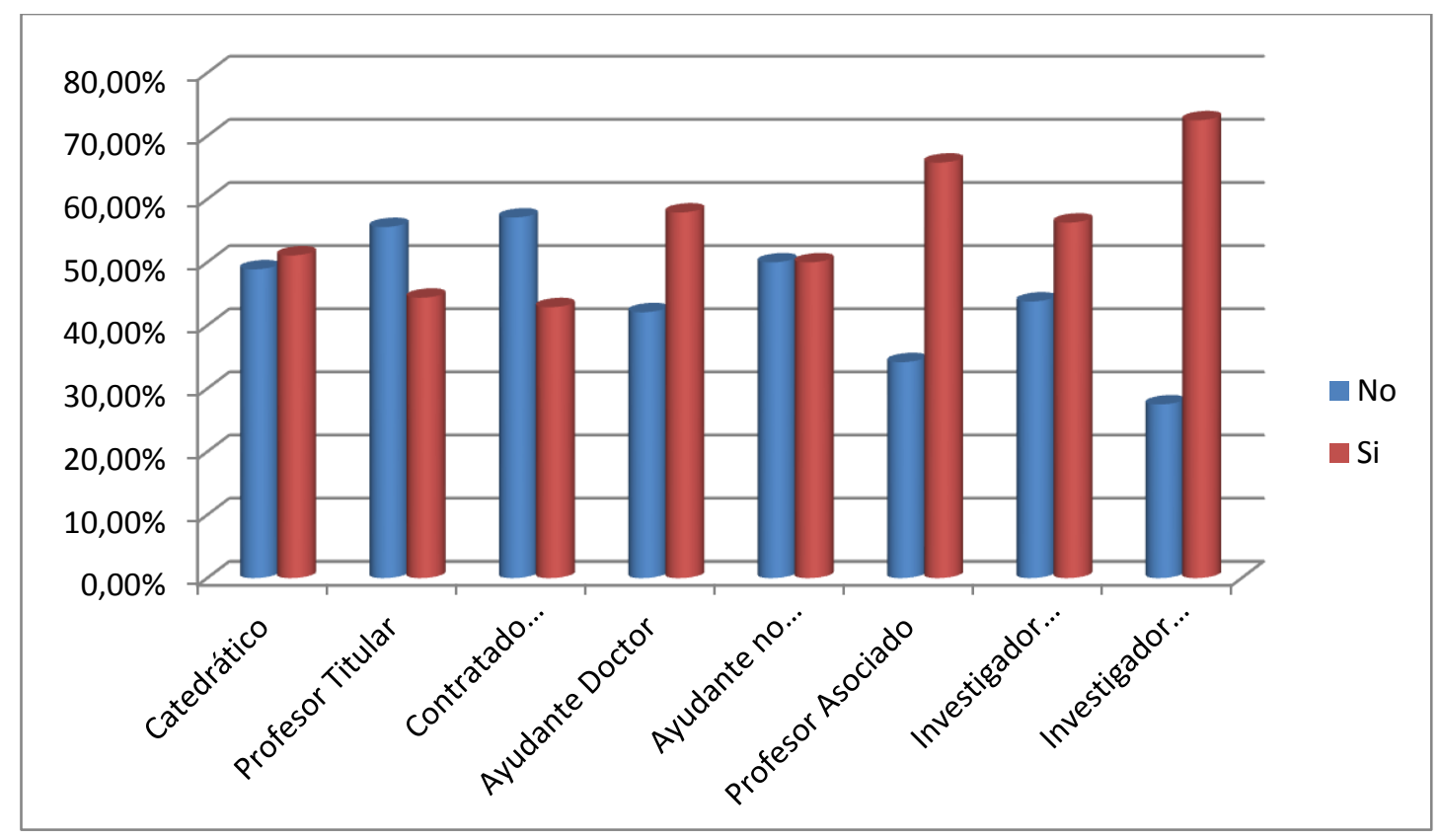

Fuente: Elaboración propia

\section{b. Utilización y valoración de buscadores}

En la encuesta también se preguntaba por el conocimiento y utilización de buscadores o aplicaciones de gestión de referencias bibliográficas por parte de los docentes e investigadores de las tres universidades gallegas. 
Los resultados muestran un alto grado de conocimiento y manejo de los buscadores. Concretamente, más de un $90 \%$ de los encuestados son usuarios de los mismos, de los cuales un 48,4\% manifiestan un dominio básico y un 43,2\% se declaran como expertos. El porcentaje de quienes declaran desconocerlos o no utilizarlos es del $6 \%$ de los encuestados. Asimismo, apenas la tercera parte $(28,73 \%)$ de los docentes e investigadores universitarios gallegos están agregados a la plataforma Orcid de identificación de autores científicos.

En concreto, Google Scholar y Scopus son los dos buscadores más conocidos, con porcentajes superiores al $80 \%$ y EBSCO y EMBASE los menos, con resultados análogos al porcentaje de utilización reconocida por los encuestados sobre los mismos, tal y como puede observarse en la siguiente tabla.

Tabla 3. Conocimiento y utilización de buscadores [2]

\begin{tabular}{|l|}
\hline Buscador \\
\hline Scopus \\
\hline Google Scholar \\
\hline Pubmed \\
\hline Medlline \\
\hline Mendeley \\
\hline Ebsco \\
\hline Embase \\
\hline Ninguno/NC
\end{tabular}

\begin{tabular}{|l|l|}
\hline Conocimiento & Utilización \\
\hline $82,29 \%$ (n 381) & $65,87 \%$ (n 305) \\
\hline $80,56 \%$ (n 373) & 64,79 (n 300) \\
\hline $32,61 \%$ (n 151) & $23,54 \%$ (n 109) \\
\hline $32,40 \%$ (n 150) & $19,65 \%$ (n 91) \\
\hline $23,54 \%$ (n 109) & $13,17 \%$ (n 61) \\
\hline $19,65 \%$ (n 91) & $10,58 \%$ (n 49) \\
\hline $4,75 \%$ (n 22) & $2,16 \%$ (n 10) \\
\hline $5,83 \%$ (n 27) & $10,80 \%$ (n 50) \\
\hline
\end{tabular}

Fuente: Elaboración propia

Por lo que respecta a la valoración de los mismos, los profesores e investigadores universitarios de las tres universidades gallegas indican una valoración media-alta en todos los casos, con una mediana de 3, siendo Scopus el único que se muestra por encima del resto, con una mediana de 4, y Embase por debajo, con una mediana de 2, tal y como consta en la siguiente tabla y gráficos.

\section{Tabla 4. Valoración de buscadores}

\begin{tabular}{|l|l|l|l|l|l|}
\hline & Media & Mediana & Desv. típ. & Min & Max \\
\hline Pubmed & 3,17 & 3,00 & 1,696 & 0 & 5 \\
\hline Medline & 3,15 & 3,00 & 1,623 & 0 & 5 \\
\hline Google Scholar & 3,39 & 3,00 & 1,093 & 0 & 5 \\
\hline Mendeley & 2,77 & 3,00 & 1,587 & 0 & 5 \\
\hline Scopus & 3,71 & 4,00 & 1,106 & 0 & 5 \\
\hline
\end{tabular}


Revista Latina de Comunicación Social\#071 - Páginas 1.187 a 1.207 [Investigación] [Financiada] | DOI: 10.4185/RLCS-2016-1141 | ISSN 1138-5820| Año 2016

\begin{tabular}{|l|l|l|l|l|l|}
\hline Embase & 2,00 & 2,00 & 1,745 & 0 & 5 \\
\hline Ebsco & 2,70 & 3,00 & 1,687 & 0 & 5 \\
\hline
\end{tabular}

Fuente: Elaboración propia

Gráfico 3. Valoración de Scopus por universidad (\%)

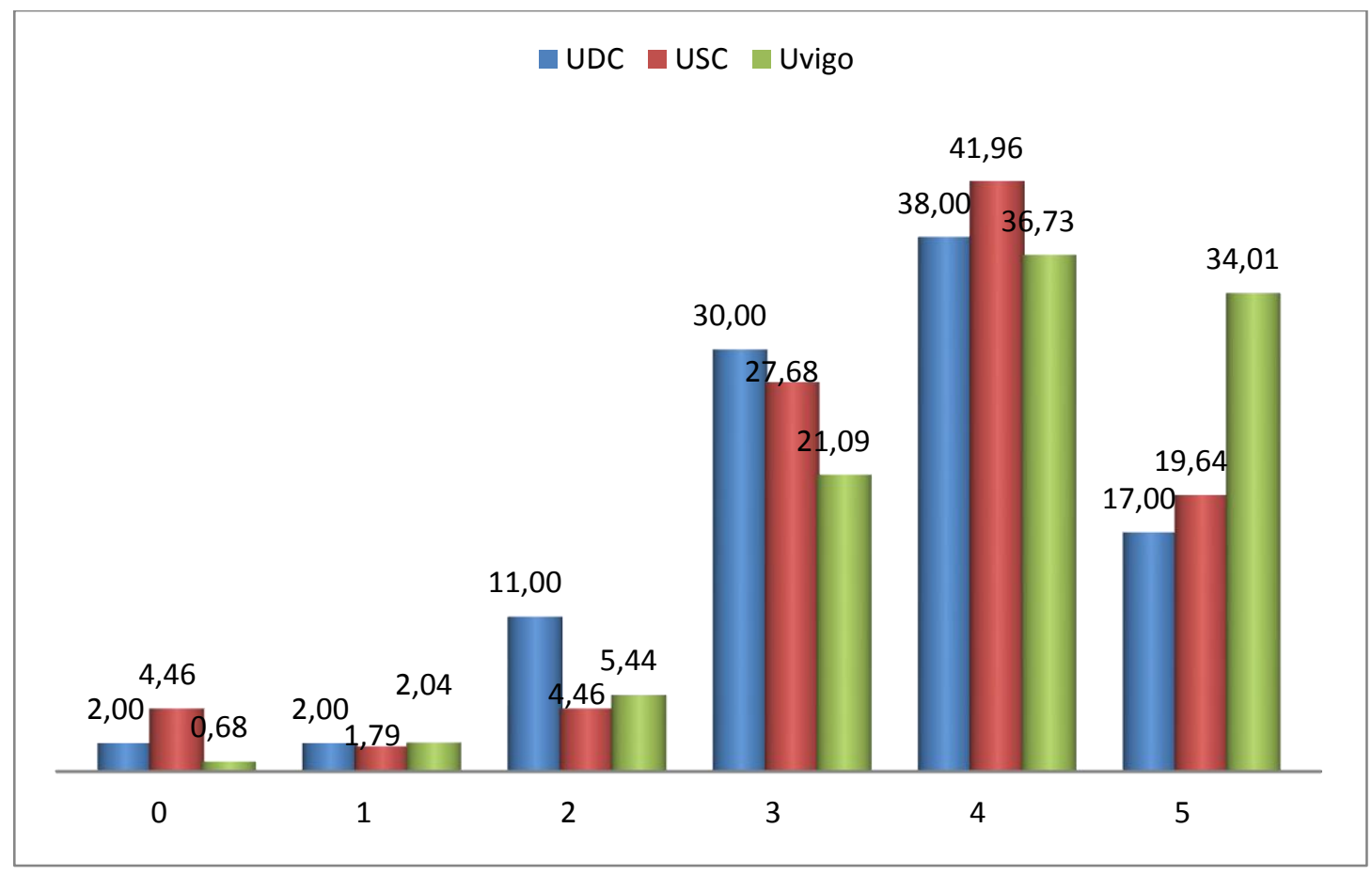

Fuente: Elaboración propia

Gráfico 4. Valoración de Google Scholar por universidad (\%)

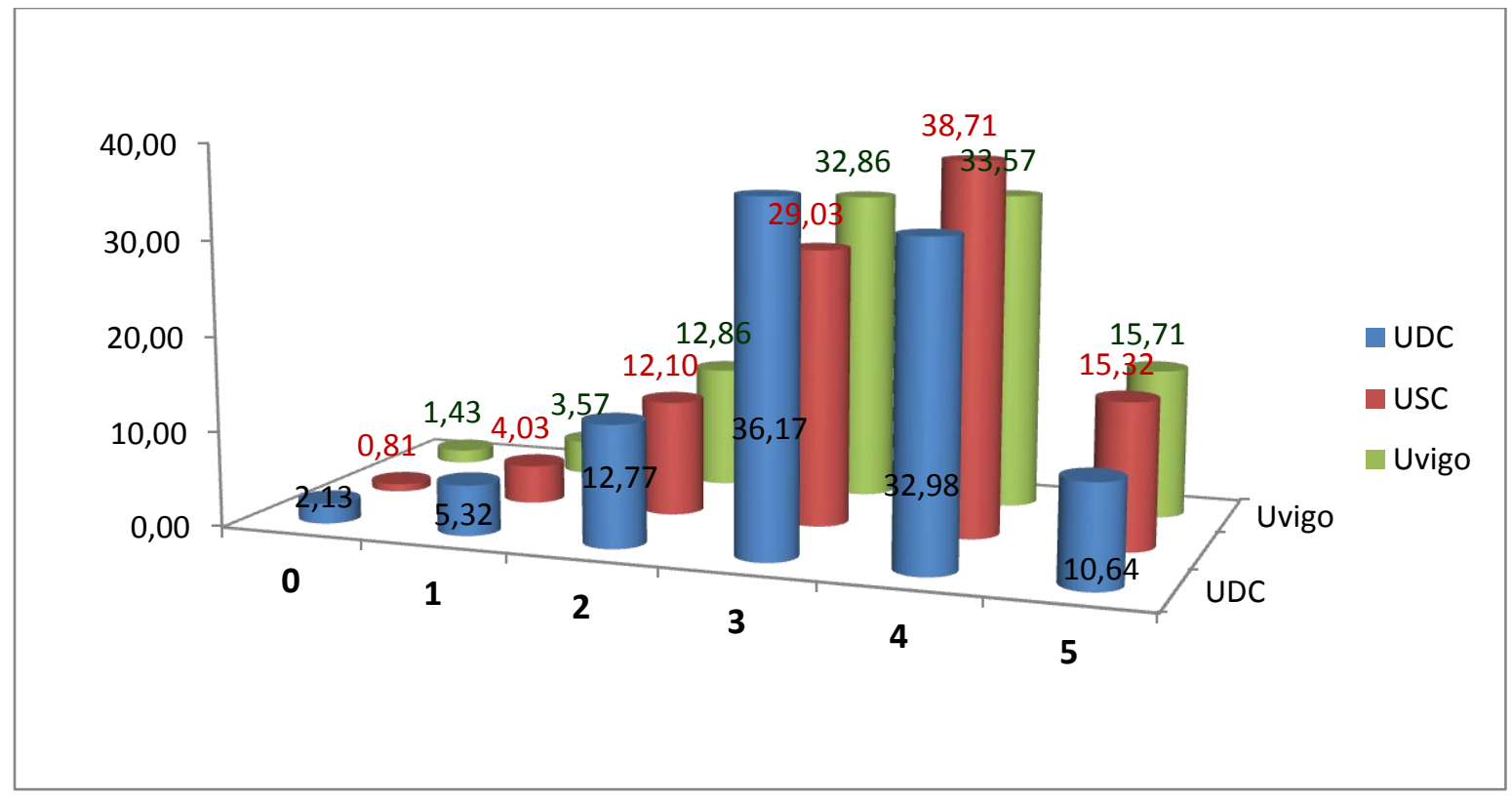

Fuente: Elaboración propia 
Revista Latina de Comunicación Social\#071 - Páginas 1.187 a 1.207 [Investigación] [Financiada] | DOI: 10.4185/RLCS-2016-1141 | ISSN 1138-5820| Año 2016

\section{Gráfico 5. Valoración de Pubmed por universidad}

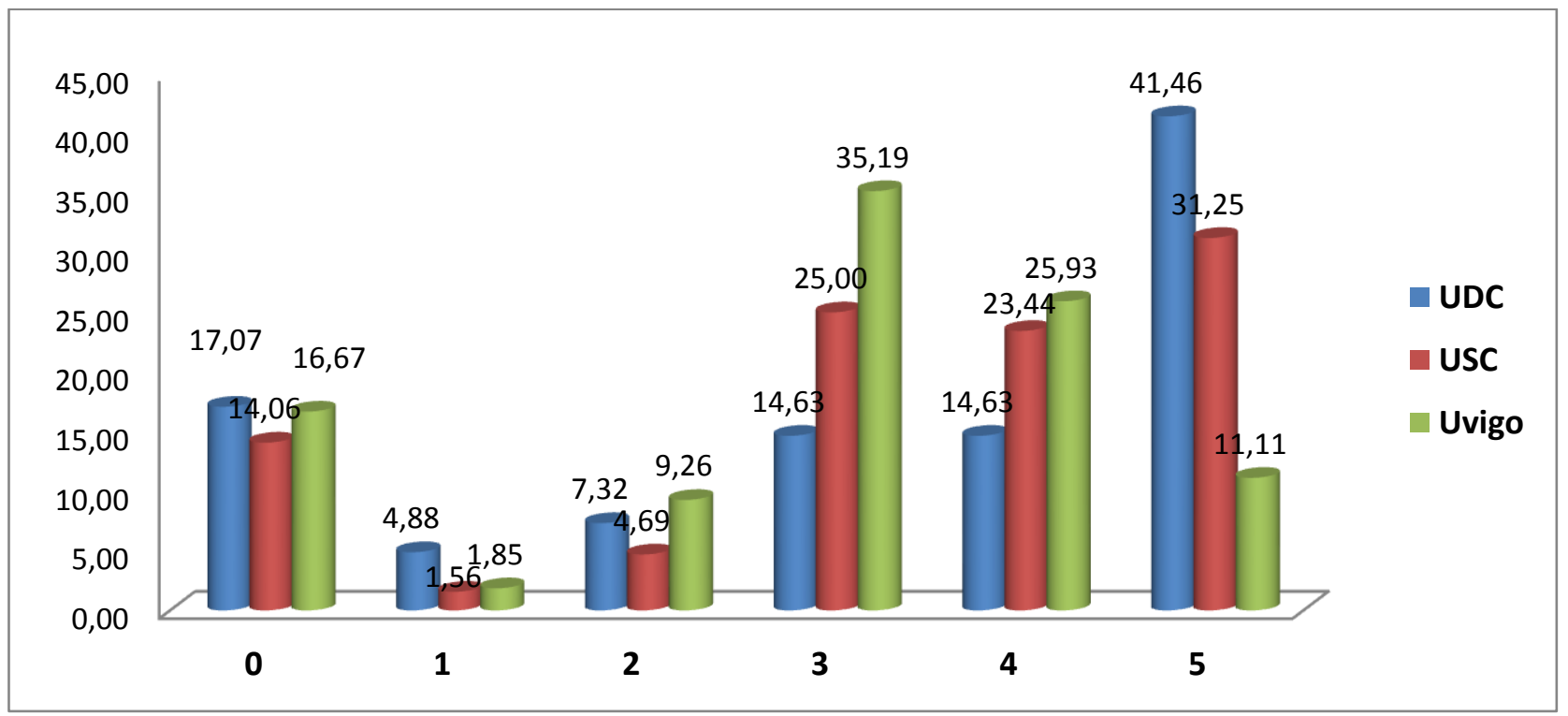

Fuente: Elaboración propia

\section{Grafico 6. Valoración de Mendeley por universidad}

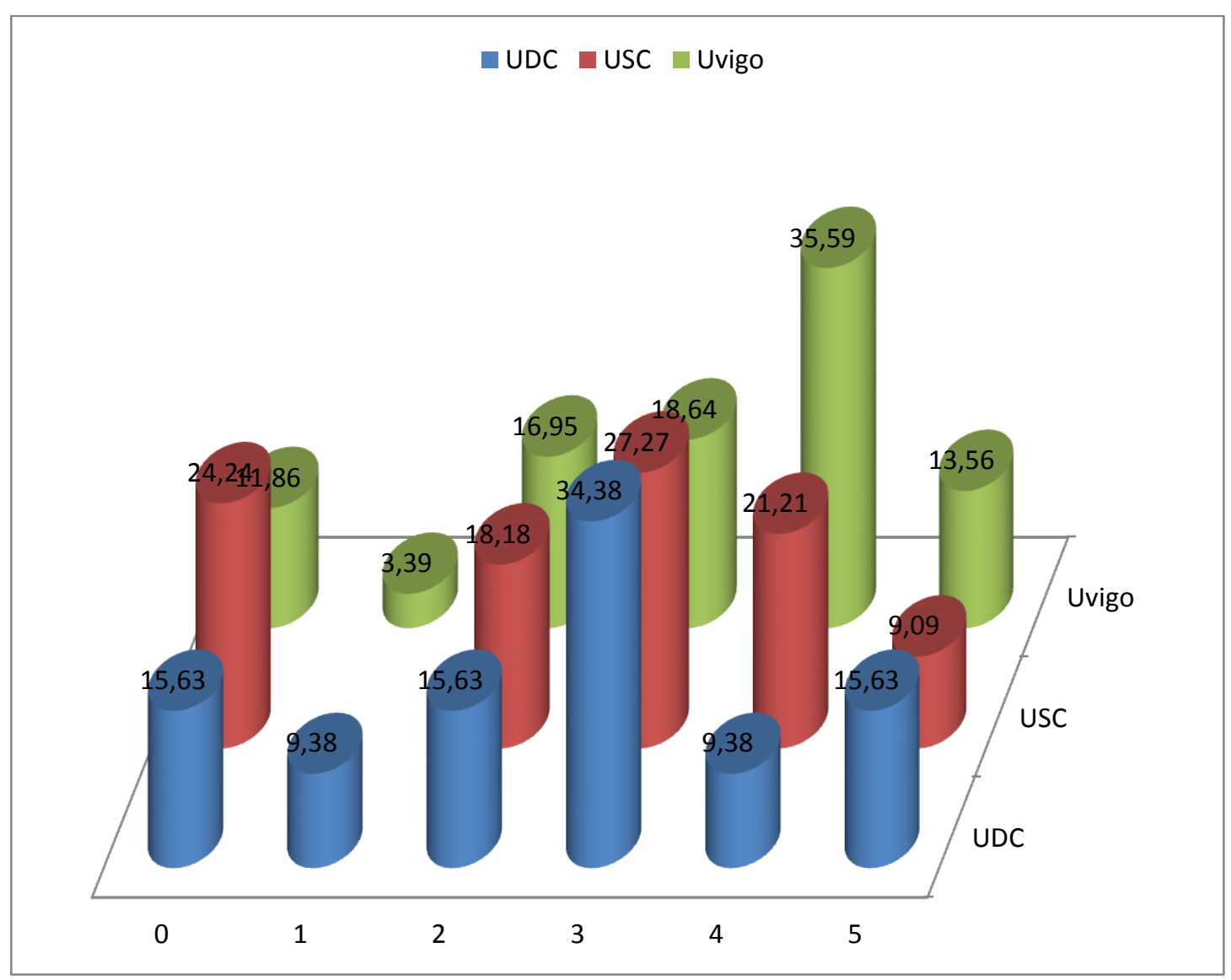

Fuente: Elaboración propia 
Finalmente, preguntados sobre qué productos de Google utiliza el PDI de las tres universidades gallegas para su actividad docente y/o científica, los resultados fueron los siguientes

Tabla 5. Productos de Google utilizados

\begin{tabular}{|l|l|l|}
\hline Producto Google & N & $\%$ \\
\hline Académico & 290 & $62,63 \%$ \\
\hline Drive & 201 & $43,41 \%$ \\
\hline Calendar & 179 & $38,66 \%$ \\
\hline Google + & 130 & $28,08 \%$ \\
\hline Groups & 40 & $8,64 \%$ \\
\hline Blogger & 27 & $5,83 \%$ \\
\hline Adwords & 9 & $1,94 \%$ \\
\hline Code & 5 & $1,08 \%$ \\
\hline NC & 69 & $14,90 \%$ \\
\hline
\end{tabular}

Fuente: Elaboración propia

\section{Discusión y conclusiones}

Los resultados de esta encuesta muestran una proporción bastante elevada y significativa en el conocimiento y utilización generalizado de buscadores en las tres universidades gallegas y opiniones bastante divididas y enfrentadas, a partes iguales, entre quienes consideran la idoneidad de las redes sociales generalistas Facebook y Twitter para la docencia y la investigación (50,2\%) y quienes no $(49,8 \%)$, con una valoración, en este último caso, más positiva a su utilización por parte del PDI menos asentado laboralmente y con menor dedicación a la universidad.

En el caso de los buscadores, la proporción entre quienes manifiestan un conocimiento y manejo básico $(48,4 \%)$ es bastante aproximada con respecto a quienes se declaran como expertos en su manejo y dominio (43,2\%). Asimismo, destacan Scopus, con un conocimiento del $82,92 \%$ entre el PDI universitario gallego y una utilización del 65,87\%, seguido de Google Scholar, con un conocimiento del $80,56 \%$ y una utilización del $64,79 \%$ ). Asimismo, se muestra un conocimiento y valoración relativos en el caso del gestor de referencias bibliográficas Mendeley, con ligeras variaciones dependiendo de cada universidad y, en cuanto a los productos de Google más utilizados para la docencia y la investigación, los más comunes son Google Académico, Drive y Calendar.

Los resultados de este trabajo contrastan, en cierta medida, con los obtenidos en una encuesta realizada por la revista Nature [3] a investigadores de distintos países sobre la utilización que hacían de las redes sociales y académicas. En la misma se preguntaba a los investigadores universitarios qué redes conocían y si las visitaban regularmente y los resultados fueron los siguientes, en orden decreciente: 1. Google Scholar, 2. ResearchGate, 3. Linkedin, 4. Facebook 5. 
Google+, 6. Twitter, 7. ResearcherID, 8. Mendeley (48\% de conocimiento y $8 \%$ de visitantes habituales) y 9. Orcid.

Como se puede observar, y pese a tratarse de un estudio realizado un año antes, ya se mostraban porcentajes superiores en el conocimiento y utilización de Mendeley que en el caso del PDI universitario gallego, pero mucho más bajos, en cambio, por lo que respecta a la plataforma ORCID, cuya utilización se observa como poco asentada, pues apenas una tercera parte $(28,73 \%)$ de los docentes e investigadores universitarios gallegas manifiestan estar agregados a la misma.

Unos datos que, sin duda, sirven para el contraste de tendencias, al igual que ocurre con la utilización de las redes generalistas, cuya utilización y reconocimiento a favor de su utilización con fines académicos, los investigadores intercalan con las restantes: Facebook en el cuarto puesto y Twitter en el sexto, esta última, aunque tan sólo es utilizada regularmente por el $13 \%$ de los encuestados, resulta más interactiva, pues el $40 \%$ de los investigadores usuarios de Twitter señalan que se trata de una buena herramienta para comentar las investigaciones que consideran relevantes para su campo.

Por otra parte, en la encuesta realizada en las tres universidades gallegas también se ofrecía la posibilidad a los encuestados de dejar algún comentario, sugerencia o crítica.

En este sentido, destacan las opiniones de quienes aprovecharon este apartado para apuntar y algún caso reiterar su escepticismo sobre la posibilidad de que redes sociales generalistas como Facebook o Twitter pudiesen contribuir, en modo alguno, a mejorar la docencia e investigación universitaria o, más bien, trivializarla, a través, por ejemplo, de la generación de chats en los que se produce una banalización de los temas. Algunos de los investigadores incluso matizan la necesidad de diferenciar, en el cuestionario aplicado, entre Facebook y Twitter, al entender que el primero es casi exclusivamente de ocio y el segundo admite varios perfiles, que pueden ser profesionales o académicos.

En cualquier caso, quedan estas consideraciones para futuras investigaciones y comparaciones con los resultados y conclusiones expuestos en este trabajo.

*Investigación financiada. Los resultados de este trabajo forman parte de los proyectos de investigación promovidos a través de la Red XESCOM (R2014/026), apoyada por la Consellería de Cultura, Educación e Ordenación Universitaria de la Xunta de Galicia; además del I+D referencia CSO2015-66543-P del Programa estatal de Fomento de la Investigación Científica y Técnica de Excelencia, subprograma estatal de Generación de Conocimiento del Ministerio de Economía y Competitividad de España; y del Programa Prometeo de la Secretaría Nacional de Educación Superior, Ciencia, Tecnología e Innovación (SENESCYT) de Ecuador, desarrollado en las Universidades Técnica Particular de Loja (UTPL) y Pontificia Universidad Católica de Ibarra (PUCESI). Los autores de esta investigación quieren agradecer a los vicerrectorados y servicios de distribución de listas de correo electrónico de las tres universidades gallegas su ayuda para la gestión de esta encuesta entre el personal docente de dichas instituciones.

\section{Notas}

1. Datos obtenidos a fecha de 21 de junio de 2016, correspondientes a las cuentas oficiales de cada una de las tres universidades. En el caso de la USC y la UVigo, constan otras dos páginas en Facebook, pero en las mismas se indica que no son oficiales (en el caso de la primera, Santiago, esa cuenta tiene incluso más likes que la oficial, 14.259, frente a 4.290. 
En el caso de UVigo las cifras están más equilibradas y domina la cuenta oficial con 7.325 me gusta, frente a los 5.500 de la no oficial). Se trata de cuentas creadas a iniciativa de los usuarios, pero sin ningún tipo de filiación o respaldo institucional.

2. Pubmed y Medline son bases de datos biomédicas, prioritariamente, y Embase, específicamente, en el campo de la medicina y farmacología. Mendeley es una red social científica que funciona también como gestor de referencias bibliográficas para la administración de citas académicas.

3. La encuesta online, realizada en agosto de 2014, recibió más de 3.500 respuestas de investigadores procedentes de 95 países. Cfr. Van Noorden, R. (2014): "Scientists and the Social Network", Nature, vol. 512, 14, pp. 126-129. Los resultados completos de la misma pueden consultarse en: http://www.nature.com/news/online-collaboration-scientists-and-thesocial-network-1.15711 (Fecha de consulta: 28 de marzo de 2016).

\section{Referencias bibliográficas}

O Almousa, (2011): "Users classification and usage-pattern identification in academic social networks". Applied Electrical Engineering and Computing Technologies (AEECT), 2011 IEEE Jordan Conference on. IEEE Conference Publications, pp. 1-6.

DOI: 10.1109/AEECT.2011.6132525

S Álvarez-García, M Gértrudix-Barrio \& M Rajas-Fernández (2014): "La construcción colaborativa de bancos de datos abiertos como instrumento de empoderamiento ciudadano". Revista Latina de Comunicación Social, 69, pp. 661-683.

DOI: 10.4185/RLCS-2014-1029. http://www.revistalatinacs.org/069/paper/1029_UR/32es.html

V Allee, (2009): "Value Creating Networks: Organizational Issues and Challenges". The Learning Organization Special Issue on Social Networks and Social Networking, vol. 6, 6, pp. 427-442. http://citeseerx.ist.psu.edu/viewdoc/download?.

JE Barker, A Johnson, L Michaelson \& B Stucky (2014): "Open Access \&amp; the Early-Career Researcher: How to use Open Access to increase your research impact". http://works.bepress.com/andrew_johnson/3/.

M Barak, A Watted \& H Haick (2016): "Motivation to learn in massive open online courses: Examining aspects of language and social engagement". Computers \& Education, 94, pp. 49-60. http://www.sciencedirect.com/science/article/pii/S0360131515300828

K Bhagat \& L Wu \& C Chang (2011): "Development and Validation of the Perception of Students Towards Online Learning (POSTOL)”. Educational Technology \& Society, vol. 19, 1, pp. 350-359.

D Boyd \& N Ellison (2007): "Social Network Sites: Definition, History and Scholarship". Journal of Computer-Mediated Communication, vol. 13, 1. http://onlinelibrary.wiley.com/doi/10.1111/j.10836101.2007.00393.x/epdf.

F Campos-Freire (2015): "Los sitios de redes sociales como paradigma del ecosistema digital". En F Campos-Freire \& J Rúas-Araujo. Las redes sociales digitales en el ecosistema mediático. Tenerife: Cuadernos Artesanos de Comunicación, 92, Sociedad Latina de Comunicación Social, SLCS. DOI: $10.4185 / \mathrm{cac} 92 \mathrm{FCF} 2015$ 
T Carney (2010): “The Third Way: Using Web 2.0 Resources in University Teaching". Valencia: 4Th International Technology, Education and Development Conference (INTED), pp. 1715-1723.

Comisión Europea (2016): “Antitrust: la Comisión envía un pliego de cargos a Google sobre el sistema operativo y las aplicaciones de Android”. Bruselas, 20 de abril. http://europa.eu/rapid/press-release_IP16-1492_es.htm.

F Charvolin, A Micoud \& L Nyhart, Lynn K. dir. (2007): Des sciencies citoyennes? París: La Tour d'Aigues, Éditions de l’Aube.

C Christensen, M Raynor \& R McDonald (2015): “What Is Disruptive Innovation?”. Harvard Business Review. https://hbr.org/2015/12/what-is-disruptive-innovation.

Crane, Diane (1972). Invisible Colleges: Diffusion of Knowledge in Scientific Communities. Chicago: University of Chicago Press.

Cheng-Jye Luh Sheng-An Yang Ting-Li Dean Huang (2016): "Estimating Google's search engine ranking function from a search engine optimization perspective", Online Information Review, vol. 40, 2 , pp. 239 - 255. http://dx.doi.org/10.1108/OIR-04-2015-0112

A Dafonte-Gómez, MI Míguez-González \& I Puentes-Rivera (2015): “Academic Social Networks: Presence and activity in Academia.edu and ResearchGate of communication researchers of the Galician universities". En: A Rocha, G Dias, A Martins, LP Reis \& M Pérez-Cota (2015). 10th Iberian Conference on Information Systems and Technologies (CISTI), Univ. Aveiro (Portugal). DOI: 10.1109/CISTI.2015.7170535. http://ieeexplore.ieee.org/xpl/articleDetails.jsp? arnumber $=7170535$.

A Dumitrache; M Gheorghe \& O Moldovan (2014): “Adapting traditional teaching techniques using new technologies". Bucarest: The International Scientific Conference eLearning and Software for Education 3: 138-141.

JM De Pablos-Coello, C Mateos-Martín \& M Túñez-López (2013): “Google cambia el paradigma de la métrica científica”. Historia y Comunicación Social, vol. 18, pp. 225-235. http://dx.doi.org/10.5209/rev HICS.2013.v18.44327.

G Eysenbach (2011): “Can Tweets Predict Citations? Metrics of Social Impact Based on Twitter and Correlation with Tradicional Metrics Scientific Impact”. Journal Internet Research, 13, 4.

DOI: $10.2196 /$ jmir.2012

V Fernández-Marcial \& L González-Solar (2015): "Promoción de la investigación e identidad digital: el caso de la Universidade da Coruña". El Profesional de la Información, v. 24, 5, pp. 656-664. http://dx.doi.org/10.3145/epi.2015.sep.14

P Flichy (2010): Le sacre de l'amateur. Sociologie des passions ordinaires à l'ère numérique. París: Seuil.

M L García Guardia y P Núñez (2010): "Nativos digitales y nuevas tecnologías: implantación en la universidad". ETD-Educação Temática Digital.

https://www.researchgate.net/publication/50993679_Nativos_digitales_y_nuevas_tecnologias_implanta cion_en_la_universidad.

A Gasparyan, NA Akazhanov \& A Voronov (2014): "Systematic and Open Identification of Resarchers and Authors: Focus on Open Researcher Contributor ID”. Journal of Korean Medical Science, vol. 29, 11, pp. 1453-1456. 
M Georgescu \& D Popescul (2014): "Social Media Literacy in Romanian Universities -are we ready yet?". En Stefan, D; Comes, C.A.; Munteanu, A; e alt. Emergin Markets Queries in Finance and Business, Procedia Economics and Finance, vol. 15, pp. 437-444.

C González-Díaz, M Iglesias-García \& L Codina (2015): "Presencia de las universidades españolas en las redes sociales digitales científicas: caso de los estudios de comunicación". El Profesional de la Información, v. 24, n. 5, pp. 640-647. http://dx.doi.org/10.3145/epi.2015.sep.12

L Gourlay (2015): "Posthuman texts: nonhuman actors, mediators and the digital university". Social Semiotics, vol. 25, n. 4, pp. 484-500.

DOI:10.1080/10350330.2015.105957

LL Haak, M Fenner \& L Paglione (2012): "ORCID: a system to uniquely identify researchers". Learned Publishing, vol. 25, 4, pp. 259-264.

Haustein, Stefanie; Peters, Isabella; Bar-Ilan, Judit; Priem, Jason; Shema, Hadas; Terliesner, Jens (2014). "Coverage and adoption of altmetrics sources in the bibliometric community". Scientometrics, vol 101, n. 2, pp.1145-1163.http://arxiv.org/abs/1304.7300.

CP Hoffman, C Lutz \& M Meckel (2015): “A reltional almetric? Network centrality on ResearchGate as an indicator of scientific impact". Journal of The Association for Information Science and Technology. DOI: $10.1002 /$ asi.23423.

HR Jamali \& M Nabavi (2015): "Open Access and sources of full-text articles in Google Scholar in different subject fields”. Scientometrics, 105, 3, pp. 1635-1651. DOI:10.1007/s11192-015-1642-2.

Jenkins, Henry; Deuze, Mark (2008). “Convergence Culture”. En Convergence: The International Journal of Research into New Media Technologies. Sage Publications, vol 14 (1), pp. 5-12.

Johnson, Thomas J.; Kaye, Barbara K. (2015). "Reasons to believe: Influence of credibility on motivations for using social networks". En Computers in Human Behavior, vol. 50, pp. 544-555. DOI: 10.1016/j.chb.2015.04.002.

H Khedmatgozar \& M Alipour-Hafezi (2015): “A basic comparative framework for evaluation of digital identifier systems". Journal of Digital Information Management, vol 13, 3.

JH Kietzmann, K Hermkens \& IP McCarthy (2011): "Social media? Get serious! Understanding the functional building blocks of social media". Business Horizons, vol. 54, 3, pp. 241-251.

Y Kim, S Jeon, Y Ji et al. (2015): "Smartphone Response System Using Twitter to Enable Effective Interaction and Improve engagement in Large Classrooms". IEEE Transactions on Education, vol. 58, 2, pp. 98-103.

M Kosemir \& D Meissner (2013): "Conceptualizing the Innovation Process - Trends and Oulook". SSRN Electronic Journal. DOI: 10.2139/SSRN.2249782.

https://www.researchgate.net/publication/236159586.

P Kothandaraman \& DT Wilson (2001): "The Future of Competition: Value-Creating Networks". Industrial Marketing Managament, vol. 30, 4, pp. 379-389.

J Kubatova (2012): "Preparing University Students for Hybrid Age - Suggestions for New Media Literacy Development". En LG Chova, AL Martínez \& IC Torres (2012): Madrid: 5Th International Conference of Education, Research and Innovation (ICERI), pp. 4866-4872. 
J Lim \& JC Richardson (2016): "Exploring the effects of students' social networking experience on social presence and perceptions of using SNSs for educational purposes". Internet and Higher Education, vol. 29, 1, pp. 31-39. DOI: 10.1016/j.iheduc.2015.12.001 .

S Manca \& M Ranieri (2016): "Facebook and the others. Potencials and obstrables of Social Media for teaching in higher education". Computers and Education, vol. 95, 1, pp. 216-230.

DOI: 10.1016/j.compedu.2016.01.012.

V Marín-Díaz, AI Vázquez-Martínez, KJ McMullin (2014): “First Steps Towards a University Social Network on Personal Learning Environments". International Review of Research in Open and Distance, vol. 15,3 , pp. $93-119$.

NA Mazov \& VN Gureev (2014): "The role of unique identifiers in bibliographic information systems". Scientific and Technical Information Processing, vol 41,3, pp. 206-210.

DOI: 10.3203/S0147688214030101.

S Mikki, M Zygmuntowska, OL Gjesdal et al. (2015): "Digital Presence of Norwegian Scholars on Academic Network Sites-Whers and Who Are They?" Plos One, vol. 10, 11, pp. 1-17.

DOI:10.1371/journal.pone.0142709

D Nicholas, E Herman, H Jamali, B Rodríguez-Bravo, Ch Boukacem-Zeghmouri, T Dobrowolski \& S Pouchot (2015): "New ways of building, showcasing, and measuring scholarly reputation". Learned Publishing, vol. 28, n. 4. http://dx.doi.org/10.1087/20150415.

BA Nosek (2012): "An Open, Large-Scale, Collaborative Effort to Estimate the Reproducibility of Psychological Science”. Psychological Science, vol. 7, n. 6, pp. 657-660.

DOI: $10.1177 / 1745691612462588$.

JL Ortega (2015): "Relationship between altmetric and bibliometric indicators across academic social sites: The case of CSIC's members". Journal of Informetrics, 9 (1), pp. 39-49.

DOI:10.1016/j.joi.2014.11.004.

J Peppard \& A Rylander (2006): "From Value Chain to Value Network: Insights for Mobile Operators". European Management Journal, vol. 24, 2-3, pp. 128-141.

N Roulin \& A Bangerter (2013): "Social Networking Websistes in Personnel Selection a Signalling Perspective on Recruiters' and Applicants'Perceptions". Journal of Personnel Psychology, vol. 12, 3, pp. 143-151.

J Sergio, L Navarro \& J Bernal (2014): "Youtube \& Facebook as Educational Tools in The TeachingLearning Process. Experience in Higher Education”. En LG Chova, AL Martínez \& IC Torres (2012): Sevilla: 7Th International Conference of Education, Research and Innovation (ICERI), pp. 940-949.

H Shema, J Bar-Ilan \& M Thelwall (2012): "Research blogs and the discussion of scholarly information". PLoS One, v. 7, n. 5. http://dx.doi.org/10.1371/journal.pone.0035869 .

JE Stigliz \& BC Greenwald (2016): La creación de una sociedad del aprendizaje. Barcelona: Planeta.

M Swijghuisen-Reigersberg (2015): "Problematizing Digital Research Evaluation using DOIs in Practice-Based Arts, Humanities and Social Science Research". F1000Research 2015, 4:193. DOI: 10.12688/f1000research.6506.1 
S Tanksalvala (2016): “As Open Access continues to grow in popularity, what is the impact?". Thomson Reuters. http://stateofinnovation.thomsonreuters.com/weighing-the-cost-and-value-of-open-access.

J Thomas, B Chen \& G Clement (2015): "ORCID Identifiers: Planned and Potential Uses by Associations, Publishers, and Librarians". The Serials Libraian: From the Printed Page to the Digital Age, vol. 68, 1-4, pp. 332-341.

DOI: $10.1080 / 0361526 X .2015 .1017713$

C Thornley, A Watkinson, D Nicholas, R Volentine, HR Hamid, E Herman, S Allard, LJ Levine \& C Carol (2015): "The role of trust and authority in the citation behaviour of researchers". Information Research-An International Electronic Journal, vol. 20, 3.

M Topaloglu, E Caldibi \& G Oge (2016): "The scale for the individual and social impact of students' social networtk use: The validity and reliability studies". Computers in Human Behavior, vol. 61, 350356.

DOI: $10.1016 /$ j.chb.2016.03.036.

M Viassone (2014): "Comunication for Educational Purposes Throughout Social Networks Sites". En D Vrontis, Y Weber \& E Tsoukatos. 7th Annual EuroMed Conference of the EuroMed-Academy of Busness, Kristiansand, Noruega.

MC Yu, Y Wu, W Alhalabi, H Kao \& WH Wu (2016): "ResearchGate: An effective altmetric indicator for active researchers?" Computers in Human Behavior, vol. 55, pp. 1001-1006.

DOI: 10.1016/j.chb.2015.11.007.

H Zhang, W Wu \& L Zhao (2016). "A study of knowledge supernetworks and network robustness in different business incubators". Physica A: Statistical Mechanics and its Applications, v. 447, pp. 545560. DOI: 10.1016/j.physa.2015.12.051.

M Zhitomirsky-Geffet \& Y Bratspiess (2015): "Perceived Effectiveness of Social Networks for Job Search". Libri, vol. 65, 2, pp. 105-118.

\section{Cómo citar este artículo / Referencia normalizada}

J Rúas-Araújo, F Campos-Freire, I Puentes-Rivera (2016): "Utilización y valoración de las redes sociales generalistas y buscadores bibliográficos en las universidades gallegas". Revista Latina de Comunicación Social, 71, pp. 1.187 a 1.207

http://www.revistalatinacs.org/071/paper/1141/61es.html DOI: $10.4185 /$ RLCS-2016-1141

\section{- En el interior de un texto:}

... J Rúas-Araújo, F Campos-Freire, I Puentes-Rivera (2016: 1.187 a 1.207)...

o ... J Rúas-Araújo et al, 2016 (1.187 a 1.207)...

Artículo recibido el 30 de julio de 2016. Aceptado el 8 de noviembre. Publicado el 12 de noviembre 